\title{
Polimedicação e doenças crônicas apresentadas por idosos de uma instituição de longa permanência
}

\author{
Polypharmacy and chronic diseases \\ presented by the elderly in a long \\ term institution
}

FisiSenectus. Unochapecó Ano 2, n. 1 - Jan/Jun. 2014 p. 25-32

Luana Cecchin . Iucecchin@hotmail.com

Acadêmica do Curso de Fisioterapia da Universidade de Passo Fundo/RS. Bolsista de Iniciação Científica PIBIC/CNPq.

Alisson Padilha de Lima . professor.alissonpadilha@gmail.com Mestrando do Programa de Pós-Graduação em Envelhecimento Humano da Universidade de Passo Fundo UPF/RS. Bolsista Pesquisador PROSUP/CAPES.

Camila Tomicki . camitomicki@gmail.com

Mestranda do Programa de Pós-Graduação em Envelhecimento Humano da Universidade de Passo Fundo UPF/RS. Bolsista Pesquisadora da Fundação de Amparo à Pesquisa do Estado do Rio Grande do Sul - FAPERGS

Marilene Rodrigues Portella .portella@upf.com

Enfermeira, Coordenadora e professora do Programa de Pós-Graduação em

Envelhecimento Humano da Universidade de Passo Fundo UPF/RS.

\section{Resumo}

Introdução: Os efeitos prejudiciais envolvendo medicamentos pela população idosa impulsionam pesquisas para desenvolvimento e aplicação de diversos métodos e instrumentos para identificar padrões inadequados de prescrição e uso. Objetivo: Avaliar a polimedicação e as doenças crônicas apresentadas por idosos de uma instituição de longa permanência. Materiais e métodos: Estudo transversal, por meio de análise de prontuários de todos os pacientes acima de 60 anos em uma instituição de longa permanência para idosos. Para a classificação dos medicamentos foram adotados os Critérios de Beers (versão 2012), que classifica os medicamentos potencialmente inapropriados para idosos. Resultados: Foram avaliados 47 idosos com média de 77,68 anos e considerouse a média de 6,32 medicamentos ao dia por idoso, sendo que destes, 55,31\% utilizavam medicamentos potencialmente inapropriados. Conclusão: Esses resultados indicam a necessidade da adoção de estratégias como a implantação de protocolos clínicos, mudanças no ensino médico e acompanhamento multiprofissional dos pacientes idosos, para evitar a automedicação e prescrição de medicamentos potencialmente inapropriados para a população idosa.

\section{Palavras-chave}

Envelhecimento, Doença crônica, Nível de saúde, Uso de medicamentos.

\section{Fisi Senectus}




\begin{abstract}
Introduction: The harmful effects of drugs involving the elderly to boost research development and application of various methods and tools to identify patterns of inappropriate prescribing and use. Objective: Assess polypharmacy and chronic diseases presented by the elderly in a long-term institution. Materials and Methods: Cross-sectional study, through analysis of medical records of all patients over 60 years in an institution for the aged, for the classification of drugs was adopted Beers Criteria 2012 version, which classifies drugs potentially inappropriate for seniors. Results: 47 seniors were evaluated averaging 77.68 years with a mean of 6.32 medications per day per individual, while $55.31 \%$ used these potentially inappropriate medications. Conclusion: These results indicate the need to adopt strategies such as the implementation of clinical protocols, changes in medical education and multidisciplinary monitoring of elderly patients to avoid self-medication and prescription of potentially inappropriate medications in the elderly population.
\end{abstract}

\title{
Keywords
}

Aging, Chronic disease, Health Status, Drug utilization.

\section{Introdução}

$\infty \times \infty \times \infty \times \infty \times \infty \times \infty \times \infty \times \infty \times \infty \times \infty \times \infty \times \infty)$

Atualmente com o aumento da população idosa e a consequente incidência de patologias que se instalam nessa fase da vida, houve um aumento expressivo da polifarmácia geriátrica ${ }^{1}$. 0 aumento da expectativa de vida se deve, em parte, às ações de promoção da saúde durante o processo de envelhecimento, e os medicamentos têm obtido papel de destaque nessa conquista ${ }^{2}$.

Com o envelhecimento, o organismo sofre alterações que o tornam mais sujeito aos efeitos tóxicos e/ou à ineficácia de certos medicamentos ${ }^{3}$. Tais alterações incluem processos farmacocinéticos, como os efeitos no sistema orgânico e nas moléculas dos medicamentos (absorção, distribuição, metabolismo e eliminação), e processos farmacodinâmicos, que é o mecanismo de ação do medicamento e seus efeitos terapêuticos, relacionando a concentração com a extensão de efeitos terapêuticos ${ }^{4}$.

Os medicamentos possuem várias funções, atuando no alívio de sintomas, cura de patologias, prevenção e diagnóstico ${ }^{5}$. No entanto, os medicamentos prescritos para pessoas idosas devem levar em consideração a relação risco-benefício para contribuir na manutenção da capacidade funcional, e não no seu comprometimento ${ }^{1}$.
Em contrapartida, alguns medicamentos são considerados potencialmente inapropriados para pessoas acima de 60 anos, devendo ser evitados pelo fato de não serem efetivos e apresentarem alto risco desnecessariamente ${ }^{3}$.

Nas Instituições de Longa Permanência para Idosos (ILPI) a preocupação com o elevado consumo de medicamentos torna-se evidente, uma vez que são consideradas unidades de saúde de baixa complexidade e que desempenham as funções de prevenção e manutenção da saúde do idoso que não possui condições de gerenciar sua própria vida ${ }^{6}$.

É identificado em pesquisa científica que idosos residentes em ILPI possuem maiores riscos para a presença de polifarmácia e de eventos adversos por apresentarem diversas patologias limitantes, fragilidade e baixa funcionalidade ${ }^{7}$.

Torna-se necessário identificar e analisar a polifarmárcia, para que tais informações possam servir de apoio para ações em saúde, possibilitando o planejamento do uso coerente de medicamentos, fornecendo subsídios para a maximização das condições de saúde individual e coletiva, bem como para a realização de projetos de cunho preventivo ou curativo ${ }^{8}$.

Desde os anos 90, alguns critérios vêm sendo propostos com o intuito de definir os medicamentos considerados poucos seguros para idosos e 
cuja prescrição deveria ser evitada nesses indivíduos, nesse contexto, os critérios sugeridos por Beers e colaboradores são os mais indicados tanto para prática clínica como na colaboração de pesquisas pertinentes ao tema⿳9. Diante desse cenário, este estudo objetivou avaliar a polimedicação e as doenças crônicas apresentadas por idosos de uma instituição de longa permanência.

\section{Materiais e métodos}

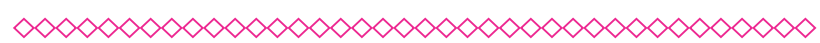

Realizou-se uma pesquisa transversal, que foi aprovada pelo Comitê de Ética em Pesquisa envolvendo seres humanos da Universidade de Passo Fundo (UPF) sob o número de protocolo 393/2011, respeitando a nova resolução 466/12, do Conselho Nacional de Saúde. A coleta de dados foi realizada em uma ILPI no município de Passo Fundo, estado do Rio grande do Sul, onde foram avaliados 47 idosos com média de idade de 77,68 anos $( \pm 10,17)$, sendo 17 do sexo masculino e 30 do sexo feminino, que apresentaram uma média de 53 meses de institucionalização.

Para serem incluídos no estudo os idosos atenderam aos seguintes critérios: idade $\geq$ há 60 anos e estar efetivamente residindo na instituição. Foram estabelecidos os seguintes critérios de exclusão: ter sofrido hospitalização no período da pesquisa.

A coleta de dados ocorreu entre os meses de maio e junho de 2014, sendo realizada por meio de avaliação das fichas pessoais e de receituários dos idosos, ambos os documentos arquivados na instituição. As informações pesquisadas foram sexo, idade, presença de doenças e medicamentos utilizados, os quais foram solicitados ao profissional de enfermagem da respectiva ILPI.

Para a classificação dos medicamentos foram adotados os Critérios de Beers versão $2012^{10}$, que classifica os Medicamentos Potencialmente Inapropriados (MPI) para idosos, independentemente da patologia, conforme as classes farmacoterapêuticas: anti-histamínicos, antiparkinsónicos, antiespasmódicos, antitrombóticos, anti-infecciosos, antagonistas adrenérgicos 1 , agonistas 2 centrais, antiarrítmicos, antidepressivos tricíclicos, antipsicóticos, barbitúricos, benzodiazepinas, hipnóti- cos não benzodiazepínicos, androgénios, sulfonilureias de longa duração, gastrointestinal, AINEs orais não seletivos das COXs e relaxantes musculares. Esse critério visa verificar se os idosos estão consumindo medicamentos inapropriados para sua faixa etária e/ou estado atual de saúde.

A estatística descritiva dos dados, os cálculos das médias e desvio padrão, a construção dos gráficos com as variáveis sociodemográficas e a relação das informações relacionadas aos medicamentos e às patologias foram realizados no programa Excel (Microsoft 2010).

\section{Resultados}

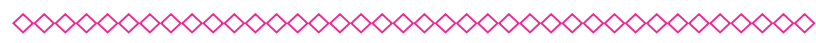

A partir dos dados obtidos pôde-se ter um escore com média de $6,32( \pm 2,67)$ medicamentos utilizados pelos idosos institucionalizados (Figura 1).

Quando avaliados os tipos de PMI, observou-se que os mais utilizados foram os benzodiazepínicos, antidepressivos tricíclicos, hipoglicemiantes e antipsicóticos, sendo que 55,31\% dos idosos utilizavam pelo menos um PMI. Identificou-se 14 tipos de fármacos PMI por meio dos prontuários apresentados na instituição, sendo o fármaco clonazepan o mais ingerido (Figura 2).

A utilização de diversos medicamentos é explicada quando associada pelas patologias identificadas por esses idosos, sendo 14 doenças identificadas pelas avaliações, destacando-se a hipertensão arterial, prevalente em 21 idosos (44,68\%), o Acidente Vascular Cerebral (AVC) identificado em 13 idosos $(27,65 \%)$ e a demência senil apresentada em $11(31,91 \%)$, (Figura 3).

\section{Discussão}

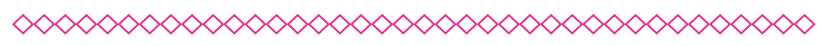

A partir dos escores obtidos pôde-se observar que os idosos consomem em média 6,34 medicamentos, entre eles o clonazepam é o mais utilizado, sendo que 55,31\% dos idosos utilizavam MPI. Tais observações vão de encontro à pesquisa de Fick et al.3, ao avaliar idosos com mais de 65 anos, que evidenciou que $54,5 \%$ a $67 \%$ das prescrições contêm pelo menos um MPI. 
Já Praxedes, Telles Filho e Pinheiro ${ }^{11}$, ao avaliarem idosos com média de idade de 78,9 anos, sendo 58,6\% mulheres, identificaram, em 55 prontuários, que $47,3 \%$ dos idosos faziam o uso de MPI, consumo consideravelmente elevado de medicamentos inapropriados.

Em outras pesquisas que avaliaram a utilização de medicamentos considerados inapropriados foram encontrados escores menores quando comparados aos do presente estudo, nessas pesquisas foram constatados índices de 33,5\%, e $28,7 \%$, respectivamente ${ }^{12-13}$.

Faustino $^{14}$ também encontrou escores menores ao avaliar a prevalência de MPI prescritos a 3.070 idosos de 60 anos ou mais, pacientes de ambulatórios de geriatria e clínica geral de um hospital universitário em São Paulo. Ao coletar as prescrições de fevereiro a maio de 2008, encontrou uma prevalência média de prescrição de MPI de $37,6 \%$ na clínica geral e $26,9 \%$ na geriatria.

Prevalência menor de utilização de MPI também apontada no estudo de Farfel et al. ${ }^{15}$ que registraram, durante seis meses, os medicamentos utilizados por idosos com 60 anos ou mais admitidos na emergência de hospital particular de São Paulo e verificaram que $19,6 \%$ deles utilizava MPI; 5,6\% receberam uma prescrição de MPI durante a permanência no departamento de emergência e outros 8,9\% tiveram alta hospitalar e foram para casa com uma prescrição inapropriada.

Escores menores também foram encontrados na pesquisa de Cassoni etal. ${ }^{16}$ que mostra, em 1.254 indivíduos com 60 anos ou mais, uma prevalência de $28 \%$ de MPI entre os idosos do Município de São Paulo, dentre estes, 83,8\% utilizavam um único medicamento potencialmente inapropriado, $13,8 \%$ usavam dois, enquanto $2,4 \%$ usavam de 3 até 5 .

Nos prontuários analisados do presente estudo foram registradas 82 prescrições medicamentosas diferentes e destas, as prescrições de medicamentos inadequados durante as internações avaliadas corresponderam aos benzodiazepínicos, antidepressivos tricíclicos, hipoglicemiantes e antipsicóticos. Estudos semelhantes também identificaram alta frequência dessas prescrições de medicamentos inadequados para idosos ${ }^{7,17}$.

Esses fármacos estão entre os medicamentos mais prescritos mundialmente. Em uma pesquisa nos Estados Unidos da América foram registrados mais de 112 milhões de receitas em 2007 e estimase que $10 \%$ a $15 \%$ da população faça uso delas 18 . Outro estudo realizado no Canadá demonstrou que o uso de benzodiazepínicos foi maior do que o uso de antipsicóticos ${ }^{19}$.

No Brasil, oSistema Nacional de Gerenciamento de Produtos Controlados da Agência Nacional de Vigilância Sanitária (Anvisa) apresentou um panorama obtido por intermédio de análises quantitativas referentes ao período de 2007 a 2010, quando foi observado que tâm ocorrido poucas alterações nos princípios ativos de maior consumo na população geral no País, sendo os principais deles o clonazepam, bromazepam e alprazolam - benzodiazepínicos que, juntos, somam mais de vinte milhões de Unidades Físicas Dispensadas (UFD) por ano20, informações que vão de encontro com esta pesquisa.

Em relação aos idosos estudados, a utilização de diferentes fármacos pode ser explicada pelas patologias apresentadas, sendo que as mais prevalentes foram à hipertensão arterial em $44,68 \%$, demência senil em $31,91 \%$ e acidente vascular cerebral em $27,65 \%$ dos idosos. Os resultados deste trabalho são comparados aos observados na pesquisa de Lucchetti et al. ${ }^{7}$ que identificaram, em 209 idosos institucionalizados, 142 com hipertensão arterial sistêmica, 77 com síndromes demenciais e 71 com acidente vascular cerebral, sendo que destes $46,4 \%$ faziam usos de cinco ou mais medicamentos concomitantemente.

Heuberger e Caudel| ${ }^{\mathbf{2 1}}$ encontraram escores maiores quando comparados a este estudo, ao avaliarem 45 idosos de uma instituição de longa permanência, sendo que destes 53,3\% apresentavam hipertensão arterial sistêmica.

Em relação à prevalência de polifarmácia nos idosos estudados no presente estudo foi de 6,34 medicamentos ao dia, sendo que esses resultados vão de encontro a um estudo brasileiro ${ }^{22}$ no qual os dados coletados com a observação de 122 idosos mostraram o uso de seis medicamentos 
regulares por idoso, sendo que 82 idosos $(67,2 \%)$ encontravam-se submetidos à polimedicação.

Em função da diversidade de doenças apresentadas pelos idosos, é comum encontrarmos a polifarmácia entre os residentes, o que é preocupante, uma vez que os efeitos da interação medicamentosa são mais acentuados nos idosos devido às alterações na absorção, metabolismo e eliminação das drogas que decorrem do envelhecimento ${ }^{23}$.

Esses resultados servem como alerta para profissionais da área de saúde e gestores de políticas públicas sobre inadequações do uso de medicamentos no âmbito dessas instituições e para evidenciar a necessidade de uma constante revisão dos esquemas terapêuticos administrados aos idosos, além de promover medidas educativas, voltadas, inclusive, para os cuidadores responsáveis pela administração dos medicamentos.

\section{Conclusão}

$\infty \times \infty \times \infty \times \infty \times \infty \times \infty \times \infty \times \infty \times \infty \times \infty \times \infty \times \infty \infty$

Os resultados encontrados na presente pesquisa indicam a necessidade da adoção de estratégias como a implantação de protocolos clínicos, mudanças no ensino médico e acompanhamento multiprofissional dos pacientes idosos, a fim de evitar a automedicação e a prescrição de medicamentos potencialmente inapropriados para a população idosa.

Cabe destacar um fator limitante para os resultados encontrados neste estudo, o fato de a pesquisa ter sido realizada em apenas uma instituição de longa permanecia, com um número pequeno de idosos internados.

Assim, estudos prospectivos tornam-se essenciais para definir mais claramente as etapas críticas do processo da polifarmácia e o uso de medicamentos potencialmente inapropriados e, dessa forma, criar ferramentas assistenciais para melhorar a qualidade da terapia farmacológica e minimizar a vulnerabilidade dos idosos aos erros de medicamentos e demais consequências da polifarmácia.

\section{Referências}

$\infty<\infty<\infty<\infty<\infty<\infty<\infty<\infty<\infty<\infty<\infty<\infty<\infty<\infty<$

1.Carvalho MFC, Romano-Lieber NS, BergstenMendes G, Secoli SR, Ribeiro E, Lebrão ML et al. Polifarmácia entre idosos do Município de São Paulo - Estudo SABE. Rev. Bras. Epidem. 2012;15(4),817-827.

2. Mazo GZ, Benedetti TB, Sacomori C. Association between participation in community groups and being more physically active among older adults from Florianopolis, Brazil. Clinics. 2011;66(11), 1861-1866.

3. Fick DM, Mion LC, Beers MH, Waller J. Health outcomes associated with potentially inappropriate medication use in older adults. Rev Nurs Health. 2008;31(1), 42-51.

4. Nóbrega OT, Karnikowski MGO. A terapia medicamentosa no idoso: cuidados na medicação. Ciênc. Saúde Coletiva. 2005;10(2), 309-313.

5. Brasil. Ministério da Saúde. Agência Nacional de Vigilância Sanitária. (2010). 0 que devemos saber sobre medicamentos. Brasília: Ministério da Saúde.

6. Brasil. Ministério da Saúde. Agência Nacional de Vigilância Sanitária. (2005) Resolução da Diretoria Colegiada n 283, de 26 de setembro de 2005. Regulamento técnico para o funcionamento das instituições de longa permanência para idosos. Brasília: Ministério da Saúde.

7. LucchettI G, Granero AL, Pires SL, Gorzoni ML. Fatores associados à polifarmácia em idosos institucionalizados. Rev. Bras. Geriatr. Gerontol. 2010;13(1), 51-58.

8. Telles Filho PCP, Almeida AGP, Pinheiro MLP. Automedicação em idosos: um problema de saúde pública. Rev Enferm. 2013;21(2),197-201.

9. Passarelli MCG. Medicamentos inapropriados para idosos: um grave problema de saúde pública. Boletim Informativo Fármaco Vigilância, 2006; (2).

10. The American Geriatrics Society 2012 Beers Criteria Update Exp ert Panel. American Geriatrics Society Updated Beers Criteria for Potentially Inappropriate Medication Use in Older Adults. J Am Soc Geriatr. 2012;60(4),616-31. 
11. Praxedes MFS, Telles Filho PCP, Pinheiro MLP. Identificação e análise de prescrições de medicamentos potencialmente inapropriados para idosos em uma instituição hospitalar. Cienc. Cuid. Saúde. 2011;10(2), 338-344.

12. Araújo CMC, Magalhães SMS, Chaimowicz F. Uso de Medicamentos Inadequados e Polifarmácia entre Idosos do Programa Saúde da Família. Rev. Lat. Am. J. Pharm. 2010;29(2), 178184.

13. Aguiar PM, Junior DPL, Silva DT, Marques TC. Avaliação de farmacoterapia de idosos residentes em instituição asilares no nordeste do Brasil. Rev. Lat. Am. J. Pharm. 2008;27(3), 454-459.

14. Idosos ambulatoriais [Dissertação de Mestrado] - Faculdade de Medicina da USP, São Paulo; 2010.

15. Farfel JM, Accorsi TAD, Franken M, Doudement SP, Moran M, Lervolino M, Bastos Neto AS. Visitas à emergência relacionadas a efeitos adversos a drogas: o papel da prescrição inapropriada. Einstein. 2010;8(2),175-79.

16. Cassoni TCJ, Corona LP, Romano-Lieber NS, Secoli SR, Duarte YAO, Lebrão ML. Uso de medicamentos potencialmente inapropriados por idosos do Município de São Paulo, Brasil: Estudo SABE. Cadernos de Saúde Pública. 2014;30(8),1708-1720.

17. Bueno CS, Oliveira KR, Berlezi EM, Eickhoff HM, Dallepiane LB, Girardon-Perlini NMO, Mafalda A. Utilização de medicamentos e risco de interações medicamentosas em idosos atendidos pelo Programa de Atenção ao Idoso da Unijuí. Rev Ciênc Farm Básica Apl. 2009;30(3), 331-338.

18. Griffin CE, Kaye AM, Bueno FR, KAYE AD. Benzodiazepine pharmacology and central nervous system-mediated effects. Ochsner Clinic. 2013;13(2), 214-23.

19. Severini SA, Dahl M, Schultz J, Metge C, Raymond C. Prescribing of psychotropic medications to the elderly population of a Canadian province: a retrospective study using administrative databases. PeerJ. 2013;10(7),168.
20. Agência Nacional de Vigilância Sanitária. Boletim de Fármaco Epidemiologia do SNGPC. 2011;1(2), 8 .

21. Heuberger RA, Caudell K. Polypharmacy and nutritional status in older adults: a crosssectional study. Drugs Aging. 2011;28(4), 315-23.

22. Fochat RC, Horsth RBO, Sette MS, Raposo NRB, Chicourel EL. Perfil de utilização de medicamentos por idosos frágeis institucionalizados na Zona da Mata Mineira, Brasil. Rev Ciênc Farm Básica Apl. 2012;33(3), 447-454.

23. Secoli SR, Figueras A, Lebrão ML, Lima FD, Santos JLF. Risk of Potential Drug-Drug Interactions among Brazilian Elderly. Drugs Aging. 2010;27(9), 759-770. 


\section{Anexos}

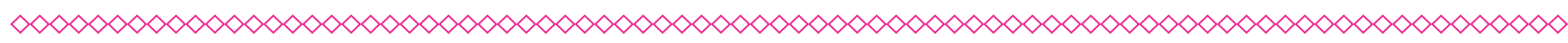

Figura 1: Quantidade de medicamentos ingeridos pelos idosos

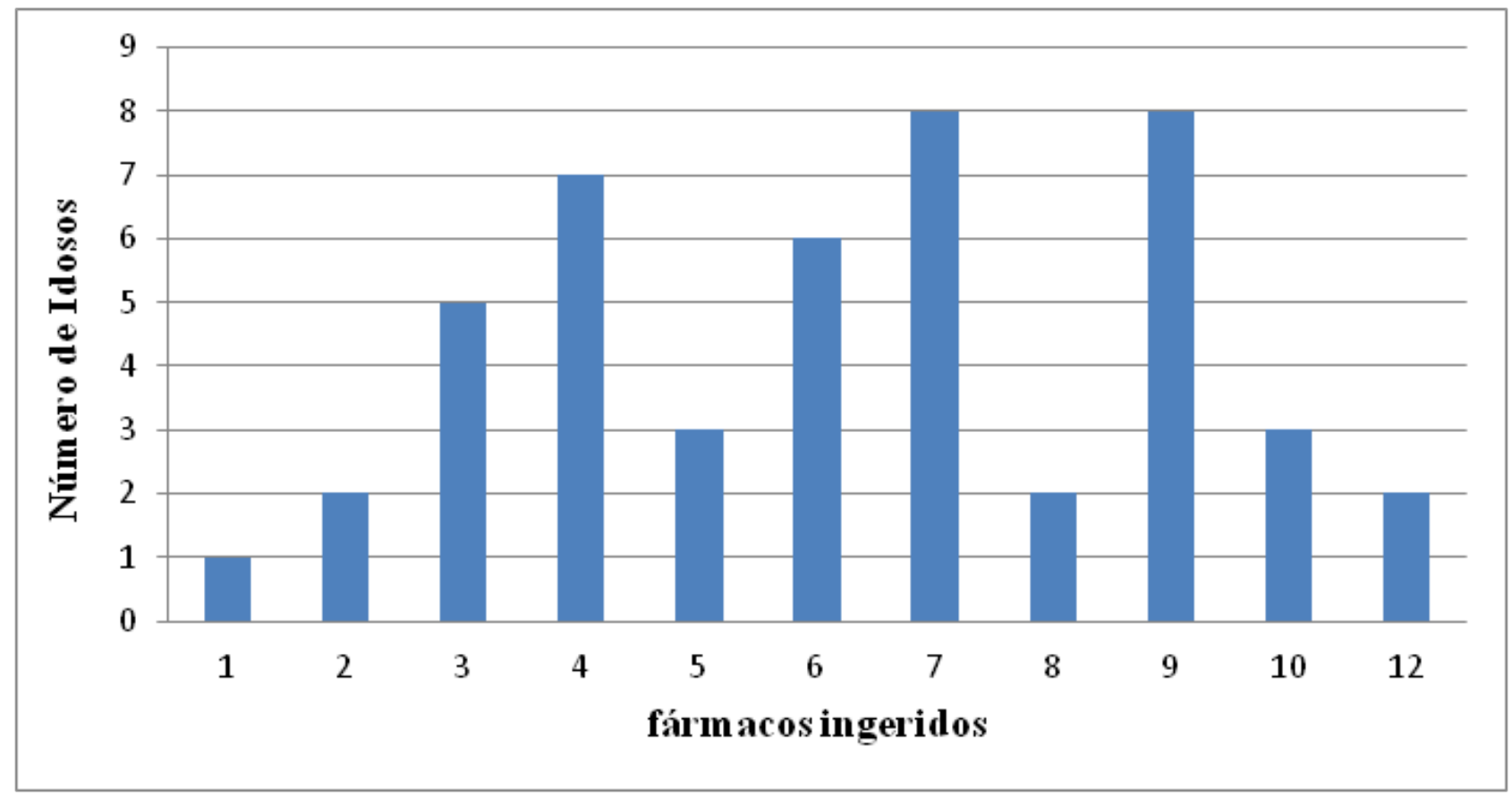

(clique para voltar ao texto)

Figura 2: Tipos de medicamentos utilizados pelos idosos

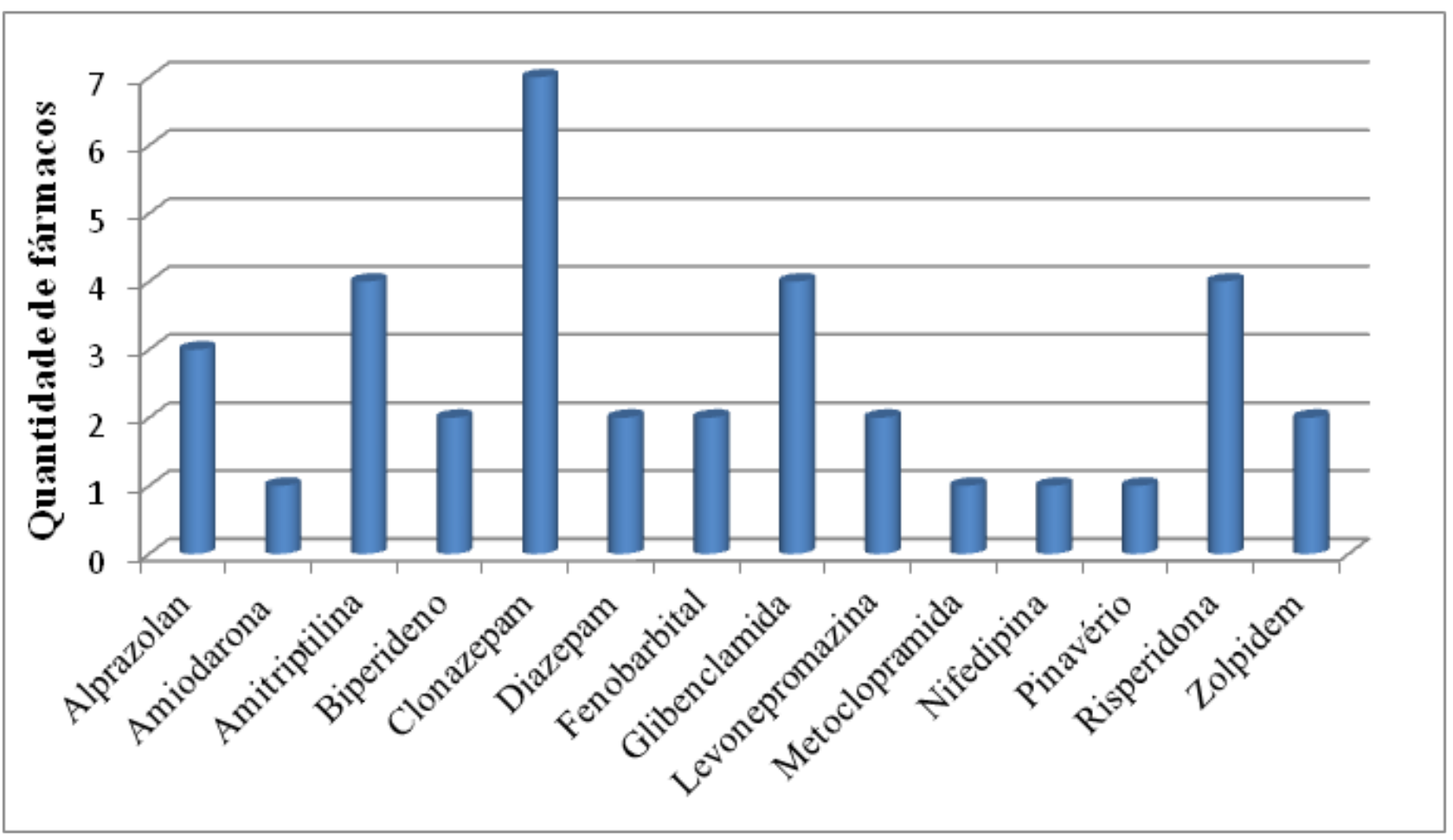

(clique para voltar ao texto) 
Figura 3: Doenças crônicas apresentadas pelos idosos

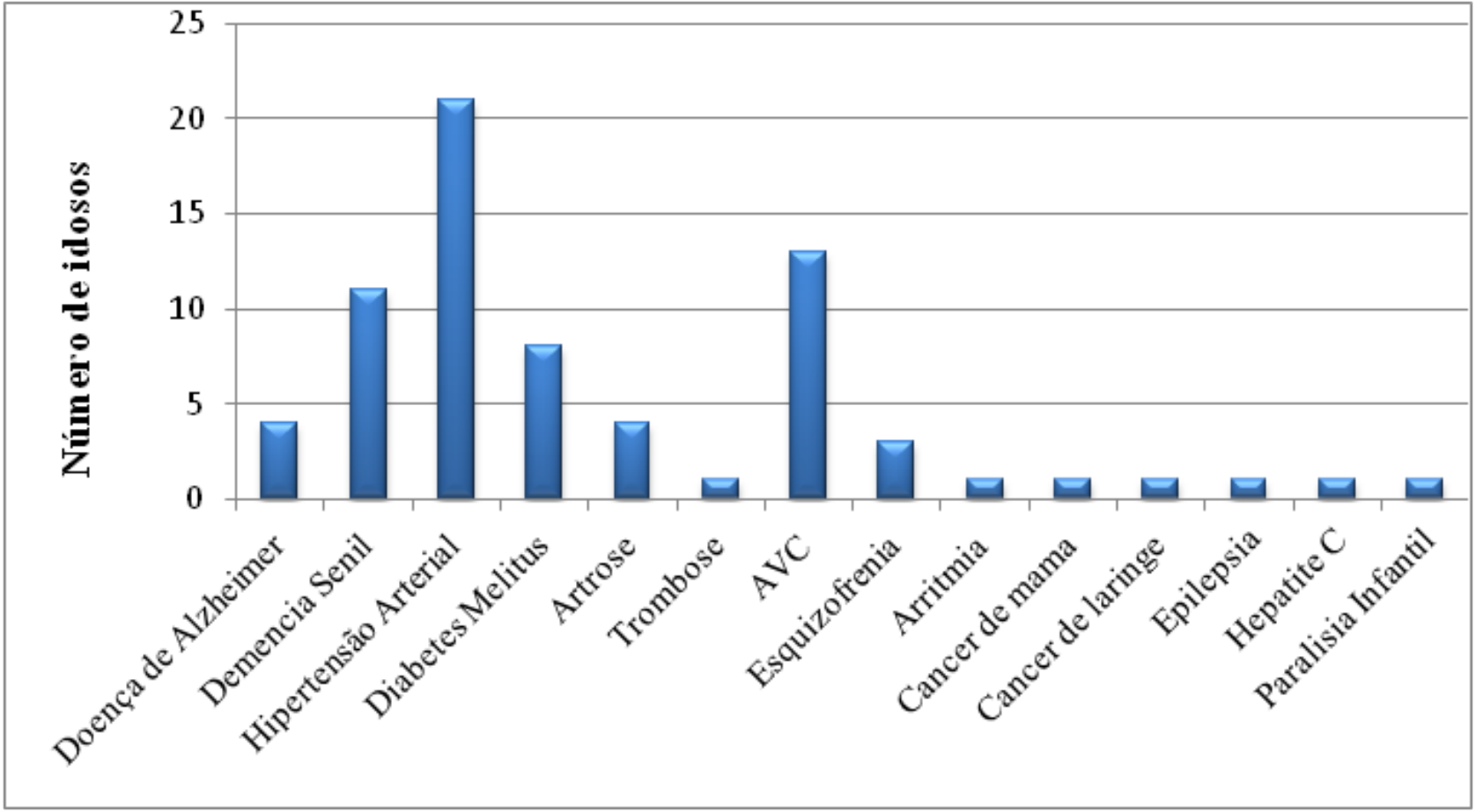

(clique para voltar ao texto) 